Check for updates

Cite this: RSC Adv., 2018, 8, 9353

Received 6th January 2018

Accepted 27th February 2018

DOI: $10.1039 / \mathrm{c} 8 \mathrm{ra00160j}$

rsc.li/rsc-advances

\section{L-Menthol alleviates cigarette smoke extract induced lung injury in rats by inhibiting oxidative stress and inflammation via nuclear factor kappa B, p38 MAPK and Nrf2 signalling pathways}

\author{
Yan Liu, ${ }^{a}$ Ang Li ${ }^{a}$ Xiuli Feng, ${ }^{a}$ Xiaoyan Jiang, ${ }^{b}$ Xiao Sun, ${ }^{a}$ Weizhen Huang, ${ }^{a}$ \\ Xiaosong Zhu ${ }^{a}$ and Zhongxi Zhao (D) *acd
}

\begin{abstract}
L-Menthol is the main ingredient of peppermint which affects various pharmacological effects such as antiinflammation and anti-oxidative activity. In this study, we aimed to evaluate the potential effects of Lmenthol on cigarette smoke extract (CSE) induced lung injury in rats. Morphology assessment results revealed that administration with L-menthol $\left(5,10\right.$ or $\left.20 \mathrm{mg} \mathrm{kg}^{-1} \mathrm{~d}^{-1}\right)$ significantly alleviated CSEinduced lung injury. Besides, L-menthol significantly reduced the inflammatory response by suppressing

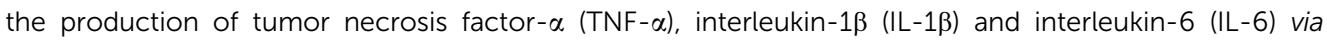
downregulating nuclear factor kappa B (NF-KB) and p38 MAPK pathways. Meanwhile, L-menthol decreased the levels of oxidative stress markers including malondialdehyde (MDA) and myeloperoxidase (MPO) whereas it increased the amount of glutathione (GSH), glutathione peroxidase (GSH-Px), superoxide dismutase (SOD) and total antioxidant capacity (T-AOC) through activation of the Nrf2 pathway. Furthermore, the expression of MMP- 9 and TIMP-1 in lungs was reduced after treatment with L-menthol, and this indicated that L-menthol might have a potential effect on airway remodeling. Moreover, immunohistochemistry analyses indicated that L-menthol could suppress the infiltration of $\mathrm{CD}^{+}$and $\mathrm{CD} 8^{+} \mathrm{T}$ cells in lung tissues and this was probably due to the immune regulation activity of $\mathrm{L}^{-}$ menthol. Taken together, our findings support that L-menthol might be a potential candidate for the treatment of CSE-induced lung injury in rats.
\end{abstract}

\section{Introduction}

Chronic obstructive pulmonary disease (COPD), as a major worldwide health problem, is characterized by persistent airflow limitation and abnormal inflammatory response in lungs. ${ }^{1}$ Cigarette smoking is regarded as the major risk factor. ${ }^{1-4}$ As is known, cigarette smoke is a complex mixture of more than 6000 chemical compounds of which oxidants are present in high concentrations. ${ }^{3}$ Briefly, cigarette smoke activates macrophages in the respiratory tract and induces the production of inflammatory cytokines and chemokine such as TNF- $\alpha$, IL-1 $\beta$, and IL-6. Then, these produced cytokines recruit inflammatory cells which could release proteases that break down connective tissue in the

${ }^{a}$ School of Pharmaceutical Sciences, Shandong University, 44 West Wenhua Road, Jinan, Shandong 250012, China

${ }^{b}$ Department of Clinical Pharmacy, Qilu Hospital, Shandong University, 107 West Wenhua Road, Jinan, Shandong 250012, China

'Shandong Engineering \& Technology Research Center for Jujube Food and Drug, 44 West Wenhua Road, Jinan, Shandong 250012, China

${ }^{d}$ Shandong Provincial Key Laboratory of Mucosal and Transdermal Drug Delivery Technologies, Shandong Academy of Pharmaceutical Sciences, 989 Xinluo Street, Jinan, Shandong 250101, China lung parenchyma and finally result in emphysema. Furthermore, this in turn causes a further release of oxidizing species and finally results in an overall redox imbalance. ${ }^{3,5,6}$ Glucocorticoid has played an essential role in the treatment of COPD. However, it can't be avoided that patients are suffering with its side effects such as steroid diabetes, osteoporosis and glucocorticoid resistance. $^{7}$

Ingredients derived from medicinal plants and natural herbal products are often regarded as promising agents due to their anti-inflammation, antioxidant and immune system function enhancing activities. Since ancient times, Mentha species have been used in traditional medicines due to their antispasmodic and carminative properties. ${ }^{8}$ Recently, more attention has been paid on the pharmacological effects of their active constituents, such as L-menthol, which is the main component of essential oil of from Mentha species. ${ }^{9}$ Several studies demonstrated that L-menthol has diverted various pharmacological properties including analgesic, ${ }^{10}$ antibacterial,,$^{11}$ antitussive, ${ }^{12,13}$ immune modulator, ${ }^{14}$ anti-apoptotic, ${ }^{15}$ antimicrobial ${ }^{16}$ and antifungal activities, ${ }^{17}$ especially its significant anti-inflammatory ${ }^{8,14,18,19}$ and antioxidative effects. ${ }^{16}$ Moreover, L-menthol was identified as the main ingredient in many compound preparations which are 
clinically effective in several respiratory diseases such as bronchitis, allergic rhinitis, laryngopharyngitis and sinusitis in China. ${ }^{\mathbf{2 0 - 2 4}}$ Taken together, L-menthol can be considered as a promising agent in the treatment of respiratory diseases.

Regarding this, we aimed to investigate the antioxidant and anti-inflammatory effects of L-menthol on cigarette smoking extract (CSE) induced lung injury in rats in the present study. Briefly, morphology assessment of lung tissues was carried out for a direct observation of its effect on lung injury. Afterwards, inflammatory mediators and the expression of related protein were analyzed. Meanwhile, the cytokine levels of antioxidant defence, oxidative stress biomarkers and relevant protein expression were examined. In addition, immunohistochemistry for the expressions of matrix metalloproteinase-9 (MMP9), tissue inhibitor of metalloproteinases-1 (TIMP-1), $\mathrm{CD}^{+}$and $\mathrm{CD}^{+} \mathrm{T}$ cells were also conducted in this work.

\section{Experimental}

\section{Materials and methods}

L-Menthol (purity of 99\%) was purchased from Sigma-Aldrich Chimie (Saint Quentin Fallavier, France). Total superoxide dismutase (T-SOD), malondialdehyde (MDA), glutathione peroxidase (GSH-Px), glutathione (GSH), myeloperoxidase (MPO), total antioxidant capacity (T-AOC), ALT and AST commercial reagent kits were obtained from Nanjing Jiancheng Biology Engineering Institute (Nanjing, Jiangsu, China). Commercial ELISA kits of TNF- $\alpha$, IL $1 \beta$ and IL-6 were purchased from Shanghai MultiSciences Biotech Co., Ltd (Shanghai, China). DAB Detection Kit was purchased from Zhongshan Goldebridge Biotechnology CO., LTD (Beijing, China). Bicinchoninic acid (BCA) kit was bought from Beyotime Institute of Biotechnology (Beijing, China). Anti-NF- $\kappa \mathrm{B}$ p65, p38, p-p38 and NQO1 antibodies were obtained from Cell Signalling Technology (Danvers, MA, USA). Anti-Nrf2 antibodies were provided by Santa Cruz Biotechnology (Santa Cruz, CA, USA). Anti-GAPDH antibodies were purchased from Proteintech Biotechnology (Rocky Hill, CT, USA). Rabbit polyclonal antibody corresponding to rat $\mathrm{CD}^{+}, \mathrm{CD} 8^{+}, \mathrm{MMP}-9$ and TIMP-1 were purchased from Abcam (Cambridge, UK). Other reagents used in the experiment were all of analytical grade.

\section{Animals}

Male SPF Sprague-Dawley rats (weight range, 120-140 g) were purchased from Laboratory Animal Center of Shandong University (Grade II, Certificate no. SCXY 20090001, Shandong, China) and were housed in individual stainless steel cages and maintained in a controlled environment (a temperature range of $22-27{ }^{\circ} \mathrm{C}$, a relative humidity of $50-70 \%$ and a $12 / 12 \mathrm{~h}$ lightdark cycle). All procedures were approved by the guidelines of the Ethical Committee Experimental Animal Center of Shandong University (no 2016020, Jinan, China). Before the formal experiment, adaptive feeding was implemented for a week.

\section{Preparation of cigarette smoke extract (CSE)}

CSE was prepared as previously described. ${ }^{25}$ In summary, three cigarettes (Taishan, China Tobacco Shandong Industrial CO. Ltd: tar, $11 \mathrm{mg}$; nicotine, $1.1 \mathrm{mg}$; carbon monoxide, $11 \mathrm{mg}$ ) were burned and the smoke was collected using a peristaltic pump, and the smoke was finally bubbled into $10 \mathrm{ml}$ of phosphatebuffered saline (PBS, pH 7.4). The CSE was freshly prepared before use and then filtered through a $0.22 \mu \mathrm{m}$ Millipore filter for removing particles and bacteria.

\section{Animal modelling}

Rat emphysema model was established by intraperitoneal injection of CSE as previously described. ${ }^{25-27}$ It was revealed that both CS exposure and intraperitoneal injection of CSE could induce rat emphysema and the effectiveness of the two different modelling methods was equal. ${ }^{25}$ However, intraperitoneal injection of CSE is more convenient in operation rather than cigarette smoke exposure daily.

Total of 42 rats were randomly divided into 7 groups $(n=6)$ : blank control, L-menthol control (20 mg kg ${ }^{-1}$ ), budesonide (positive control, $10 \mathrm{mg} \mathrm{kg}^{-1}$ ), CSE, and L-menthol (5 $\mathrm{mg} \mathrm{kg}^{-1}$, $10 \mathrm{mg} \mathrm{kg}^{-1}$ and $\left.20 \mathrm{mg} \mathrm{kg}^{-1}\right)$. The treatment doses of L-menthol were selected based on our preliminary study. Rats in CSE group were intraperitoneal injected with $1 \mathrm{ml}$ CSE-PBS solution on days 1, 8, and 15. Animals from budesonide and L-menthol groups were injected intraperitoneally with budesonide $(10 \mathrm{mg}$ $\left.\mathrm{kg}^{-1} \mathrm{~d}^{-1}\right)$ and $\mathrm{L}$-menthol (5, 10 and $\left.20 \mathrm{mg} \mathrm{kg}^{-1} \mathrm{~d}^{-1}\right)$ daily for 21 days, along with intraperitoneal injection of CSE on days 1, 8, and 15 the same as CSE group. Rats from L-menthol control group were administrated with L-menthol $\left(20 \mathrm{mg} \mathrm{kg}^{-1}\right)$ daily for evaluating its effect on normal rats without the administration of CSE. L-Menthol and budesonide solutions were fresh prepared by dissolving them in the injection vehicle of PBS containing $20 \%$ hydroxypropyl- $\beta$-cyclodextrin (w/v). Rats from the blank control group received intraperitoneal injection of PBS instead of CSE or drugs. Rats were all fed under the same rearing condition for 21 days and were sacrificed on the 21st day after the experiment.

\section{Bronchoalveolar lavage fluid (BALF) preparation and lung tissue homogenization}

Rats were intraperitoneal injected with $10 \%$ chloral hydrate $\left(3 \mathrm{ml} \mathrm{kg}^{-1}\right.$ ) for euthanasia. Bronchoalveolar lavage was performed on the right lung while the left lung was clamped for further preparation.

The trachea and the right lung were cannulated and perfused with $2 \mathrm{ml}$ ice cold normal saline for 3 times. Then the BALF was merged and the total volumes of BALF were recorded. After immediately centrifuged for $10 \mathrm{~min}$ at $1500 \mathrm{rpm}$ and $4{ }^{\circ} \mathrm{C}$, pelleted cells were resuspended with normal saline. The total number of cells was counted using a hemocytometer while differential cell counts were stained with Wright-Giemsa stain according to the manufacturer's instructions (Beyotime Institute of Biotechnology, China). Total of 400 leukocytes were counted on each slide and measured by high microscopy at 
a magnification of $\times 1000$. The percentages of macrophage, neutrophil and lymphocyte in BALF were calculated after differential cell counts. The left lungs were subsequently homogenized in ice normal saline for preparing the lung homogenates $(10 \%, w / v)$ and centrifuged for $10 \mathrm{~min}$ at $3500 \mathrm{rpm}$ and $4{ }^{\circ} \mathrm{C}$. Contents of protein in the supernatant were determined using bicinchoninic acid (BCA) method. Samples were all stored in a freezer $\left(-80^{\circ} \mathrm{C}\right)$ until biochemical analyses.

\section{Morphology assessment of lung tissues}

For histologic analysis, the left upper lobes were fixed in $10 \%(\mathrm{v} / \mathrm{v})$ neutral buffered formalin and then embedded in paraffin. Paraffin sections of lung tissues were stained with hematoxylin and eosin (HE) solution after sectioned at $4 \mu \mathrm{m}$ thickness. The mean linear intercept (MLI) and destructive index (DI) were determined in lung tissues as described before. ${ }^{28,29}$

Mean linear intercept (MLI) is a measurement of the mean inter alveolar septal wall distance to assess the airspace enlargement, and is widely used to indicate the average size of alveoli. MLI was assessed in 10 random and non-overlapping fields per lung by light microscopy at a $100 \times$ magnification. Cross lines were drawn in a field excluding vessels and bronchus and the total number of alveolar septa of the two lines was counted. The total length of the cross lines divided by the number of intercepts gives the MLI, that is, MLI = total length/ number of alveolar septa, as described previously. ${ }^{28,29}$

Destructive index (DI) is used for indicating the percentage of destroyed alveoli and for estimating of lung parenchymal destruction. DI is quantified by dividing the number of destroyed alveoli by the total number of the counted alveoli. Alveoli lying underneath the counting points were evaluated for the presence of destruction. Destructive alveolus was defined if at least one of the following alveoli conditions was observed: at least 2 alveolar wall defects, at least 2 intraluminal parenchymal rags in alveolar ducts, clearly abnormal morphology, or classic emphysematous changes. ${ }^{28,29}$

\section{Measurement of inflammatory cytokines using enzyme linked immuno sorbent assay (ELISA)}

The levels of TNF- $\alpha$, IL- $1 \beta$ and IL- 6 in the lung homogenates were detected using commercial ELISA kits according to the manufacturers' protocols.

\section{Determination of oxidative stress biomarkers in lung homogenate}

The contents of glutathione (GSH), glutathione peroxidase (GSH-Px), superoxide dismutase (SOD), total antioxidant capacity (T-AOC), malondialdehyde (MDA) and myeloperoxidase (MPO) in the lung homogenates were measured according to the guidelines of the commercial assay kits.

\section{Western blotting analysis for expression of proteins}

The lung tissues were homogenized in ice-cold radio immunoprecipitation assay (RIPA) lysis buffer (SolarbioLife Sciences) which contained $1 \mathrm{mmol} \quad \mathrm{L}^{-1}$ of phenylmethylsulfonyl fluoride (PMSF, Sigma Aldrich, St. Louis, MO, USA) and 1\% cocktail protease inhibitors (Sigma). Proteins were extracted by centrifugation and their contents were determined using the BCA method. Briefly, a total of 50 $\mu \mathrm{g}$ of proteins were separated on sodium dodecyl sulfate polyacrylamide gel electrophoresis (SDS-PAGE) and then transferred onto the nitrocellulose (NC) membranes. The membranes were subsequently incubated with blocking solution (5\% skim milk) in TBST buffer for $1 \mathrm{~h}$. Following blocking, these membranes were washed with TBST buffer and incubated with specific antibodies against NF-кB p65, p38, p-p38, Nrf2, NQO1 $(1: 1000)$ and anti-GAPDH $(1: 3000)$ over night at $4{ }^{\circ} \mathrm{C}$. After thoroughly washed with TBST buffer for 3 times, these membranes were incubated with horseradish peroxidase (HRP)-conjugated antibodies (1:5000) for $2 \mathrm{~h}$ at room temperature. Finally, enhanced chemiluminescence (ECL, Merck Millipore, Darmstadt, Germany) detection was conducted with the membranes after thoroughly washed with TBST buffer. The quantification of bands was carried out using the Alphalmager HP system (Cell Biosciences, Inc., Santa Clara, CA, USA). AlphaView SA software (Cell Biosciences, Inc., Santa Clara, CA, USA) was adopted for quantifying the relative optical densities of the bands. All western blot analyses were carried out in triple.

\section{Immunohistochemistry for expression of $\mathrm{CD}^{+}, \mathrm{CD8}^{+}, \mathrm{MMP}-9$ and TIMP-1}

The immunohistochemistry assays were conducted for determining the expression levels of $\mathrm{CD} 4^{+}, \mathrm{CD} 8^{+}$, MMP-9 and TIMP-1 in lungs. The primary antibodies were rabbit polyclonal antibody corresponding to rat $\mathrm{CD}^{+}, \mathrm{CD}^{+}$, MMP-9 and TIMP-1 $(1: 200)$. Immunohistochemistry assays were conducted according to the manufacturers' instructions. Briefly, paraffins which were embedded with lung tissue slice were deparaffinized and then rehydrated. Subsequently, antigen was retrieved using sodium citrate and with heat-induced retrieval. After blocking with goat serum, anti-CD $4^{+}, \mathrm{CD}^{+}$, MMP-9 and TIMP-1 antibodies were applied overnight at $4{ }^{\circ} \mathrm{C}$, respectively. Then, horseradish peroxidase-conjugated second antibodies were applied, and the expression of $\mathrm{CD}^{+}, \mathrm{CD} 8+$, MMP-9 and TIMP-1 were finally visualized using a DAB detection kit. Positive expressions of $\mathrm{CD}^{+}, \mathrm{CD}^{+}$, MMP-9 and TIMP-1 were shown to be brown. Images of $\mathrm{CD}^{+}, \mathrm{CD}^{+}$, MMP-9 and TIMP-1 were obtained and photographed under microscope.

\section{Statistical analysis}

Graphpad Prism version 5.0 (Graphpad Prism Software, San Diego, CA, USA) was adopted for performing all the statistical analysis. Data are presented as mean \pm SEM. Comparisons between two groups were conducted with an unpaired twotailed Student's $t$-test. Multiple groups comparison were performed with analysis of variance (ANOVA) followed by $t$-test with the Bonferroni adjustment. A $p$-value less than 0.05 was considered statistically significant. 


\section{Results}

\section{L-Menthol attenuated CSE-induced lung histopathologic changes}

Morphometric assessments of the lung parenchyma were performed in order to evaluate whether administration of L-menthol could reduce CSE-induced lung injury. Previous study indicated that intraperitoneal injection of CSE was a new convenient method for rat emphysema modelling, which showed no significant difference from typical cigarette smoking exposure method. ${ }^{25}$ All HE stained lung tissue slices were observed under light microscope $(100 \times)$. As shown in Fig. 1, lung tissues of the blank control and L-menthol control group showed normal pulmonary histology (Fig. 1A(I and II)), whereas lung tissues of CS group (Fig. 1A(III)) revealed significantly histopathology changes including enlargement of alveolar air spaces and destruction of lung parenchyma. On the contrary, the alleviation of lung injury in both budesonide (10 $\mathrm{mg} \mathrm{kg}^{-1}$, Fig. 1A(IV)) and L-menthol groups (5, 10, $20 \mathrm{mg}$ $\mathrm{kg}^{-1}$, Fig. 1A(V-VII)) were found, with decreased values of MLI (Fig. 1B) and DI (Fig. 1C) $(p<0.001)$.

\section{Effect of L-menthol on body weight and spleen/liver index}

As shown in Fig. 2A, the body weights of rats in CSE and budesonide groups were markedly decreased compared to blank control group $(p<0.01)$, whereas there was no significant difference between CSE and budesonide group ( $p>0.05)$. However, greater values of body weights of rats were found in three L-menthol treated groups compared to CSE group ( $p<$ 0.001). Moreover, the difference of body weights among blank control, L-menthol control and three L-menthol treated groups were not statistically significant $(p>0.05)$.

As previously reported, spleen index is partly in connection with immune function and can be used for evaluating immune function preliminary. ${ }^{30}$ In this study, a significant decrease of spleen index was observed after treated with budesonide compared to CSE group (Fig. 2B) $(p<0.001)$ whereas the values of rats from three L-menthol groups were markedly increased with a dose dependent manner $(p<0.001)$. Besides, it's worth noting that a decrease of liver index of rats was appeared after treatment with CSE $(p<0.001)$ (Fig. 2C). Moreover, the values of liver index of rats from budesonide group were much lower than that in CSE group $(p<0.001)$ (Fig. 2C). Conversely, the treatment with L-menthol increased the liver index compared to CSE group $(p<0.001)$. Besides, aspartate aminotransferase (AST) and alanine aminotransferase (ALT) are sensitive biochemical markers for hepatic damage. Our results indicated that the production of AST (Fig. 2D) and ALT (Fig. 2E) were obviously enhanced after treated with $\operatorname{CSE}(p<0.01)$. However, budesonide could not reduce the levels of AST and ALT, but resulting in higher production $(p<0.05$ or $p<0.01)$. Conversely, the levels of ALT and AST were markedly reduced in three L-menthol groups

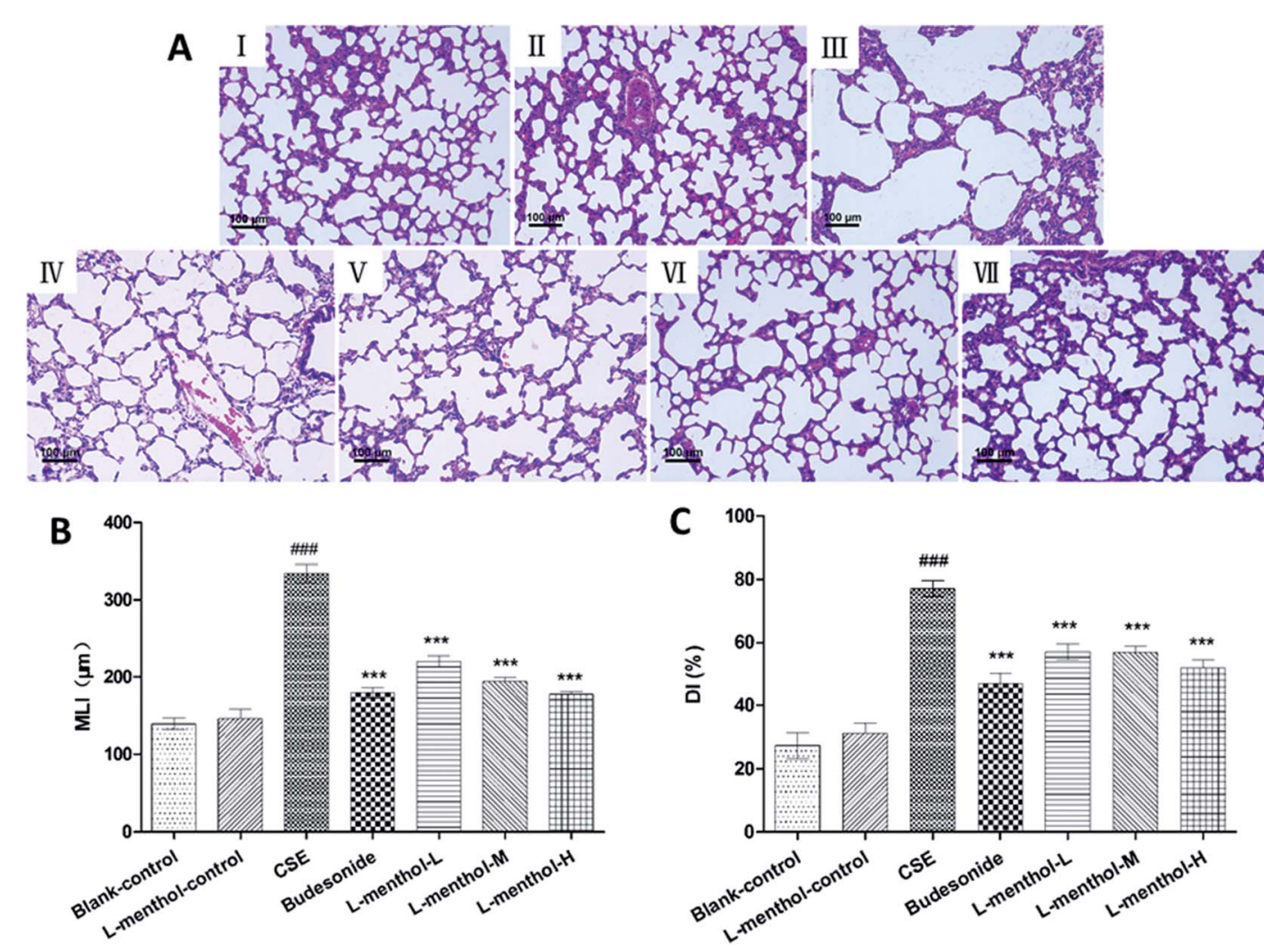

Fig. 1 Effect of L-menthol on histological changes in lung tissues of CSE-induced lung injury in rats (100 $\times$ magnification) (A). Blank control group (I); L-menthol control group (20 mg kg $\mathrm{m}^{-1}$ ) (II); CSE group (III); budesonide group (10 mg kg $\mathrm{d}^{-1}$ ) (IV); L-menthol-L group (5 mg kg $\mathrm{kg}^{-1}$ ) (V); L-menthol-M group (10 mg kg $\mathrm{d}^{-1}$ ) (VI) and L-menthol-H group (20 $\left.\mathrm{mg} \mathrm{kg}^{-1} \mathrm{~d}^{-1}\right)$ (VII). Morphometric measurements of mean linear intercept $(\mathrm{MLI})(\mu \mathrm{m})(\mathrm{B})$ and destructive index (DI) (\%) (C). ${ }^{\# \#} p<0.001 \mathrm{vs}$. the blank control group. ***p 0.001 vs. the CSE group. 



Fig. 2 Effect of L-menthol on body weights (A), spleen index (B), liver index (C), ALT (D) and AST (E) of rats in all groups. (A) Rat body weights were measured in each group at the indicated time point. (B) The spleen index of rats in each group. Weight (spleen) $\mathrm{g} \times 10 /$ weight (body) $\mathrm{kg}$. (C) The liver index of rats in each group. Weight (liver) g/weight (body) kg. Data are presented as mean \pm SEM $(n=6 /$ group). $\# \# \# p<0.001$ vs. the blank control group. ${ }^{*} p<0.05,{ }^{*} p<0.01$ and ${ }^{* * *} p<0.001$ vs. the CSE group. ${ }^{\circ} p<0.01$ and ${ }^{\circ 00} p<0.001$ vs. the budesonide group.

$(p<0.001)$. Taken together, it was demonstrated that $\mathrm{L}-\mathrm{menthol}$ might prominently ameliorate CSE-induced liver injury.

\section{Effect of L-menthol on cellular influx in BALF}

Previous finding showed that macrophages, neutrophils and lymphocytes have played essential roles in chronic immune inflammation in COPD which are mainly induced by CSE. ${ }^{31}$ In this regard, the total numbers of white blood cells and differential counts of neutrophils, macrophages and lymphocytes in BALF were determined. As shown in Fig. 3A, a nearly 3 fold increase of total numbers of white blood cells was observed in CSE group compared to blank control group $(p<0.001)$. In addition, differential counts of neutrophils, macrophages and lymphocytes revealed that macrophages in BALF of CSE group were obviously increased whereas the numbers of total white blood cells and macrophages were markedly decreased in budesonide and $\mathrm{L}$-menthol treated groups $(p<0.05$ or $p<0.001)$.

\section{L-Menthol suppressed CSE-induced inflammatory cytokines}

Tumor necrosis factor- $\alpha$ (TNF- $\alpha$ ), interleukin-1 $\beta$ (IL-1 $\beta$ ) and interleukin-6 (IL-6) are the important pro-inflammatory cytokines which can be induced by CSE. ${ }^{5}$ The levels of TNF$\alpha$, IL-1 $\beta$ and IL- 6 in lung parenchymal were evaluated in order to investigate the anti-inflammation effect of $\mathrm{L}$-menthol (Fig. 3B-D). Briefly, the obviously higher productions of these cytokines were observed in CSE group compared with the blank control group $(p<0.001)$ whereas treated with budesonide and L-menthol could significantly reduce their levels $(p<0.05, p<0.01$ or $p<0.001)$ relative to CSE group. In summary, these results demonstrated that L-menthol could 



Fig. 3 Effect of L-menthol on cell influx in bronchoalveolar lavage fluid (BALF) (A), tumor necrosis factor- $\alpha$ (TNF- $\alpha)(B)$, interleukin-1 $\beta$ (IL-1 $\beta)$ (C) and interleukin-6 (IL-6) (D). Data are presented as mean \pm SEM ( $n=6 /$ group). ${ }^{*} p<0.05,{ }^{\# \#} p<0.01$ and ${ }^{\# \# \# ~} p<0.001$ vs. the blank control group. $* p<0.05, * * p<0.01$ and $* * * p<0.001$ vs. the CSE group. ${ }^{\circ} p<0.05,{ }^{\circ}{ }^{\circ} p<0.01$ and ${ }^{\circ 0 \circ} p<0.001$ vs. the budesonide group.

alleviate airway inflammation by suppressing proinflammatory in CSE treated rats.

\section{Effects of $\mathrm{L}-\mathrm{menthol}$ on oxidative stress markers in lung parenchyma}

MDA functions as an indicator of oxidative stress which is correlated inversely with pulmonary function while MPO could increase the amount of ROS and finally result in the irreversible lung damage. ${ }^{32-34}$ In the present study, MPO and MDA levels in CSE group were obviously increased compared to blank control group $(p<0.001)$ whereas lower MPO and MDA levels were observed in budesonide and L-menthol treated groups $(p<0.05$ or $p<0.001$ ) (Fig. $4 \mathrm{~A}$ and $\mathrm{B}$ ).

GSH and GSH-Px are important antioxidants for protecting airway epithelium from oxidant damage while SOD functions as a scavenger of superoxide radical. ${ }^{33,35}$ As shown, GSH, GSH-Px, SOD and T-AOC levels were markedly decreased in rats from CSE group relative to blank control group ( $p<0.001$ ) (Fig. 4C-F). Inversely, rats from L-menthol and budesonide groups showed higher levels of GSH, GSH-Px, SOD and T-AOC than that in CSE group $(p<0.05, p<0.01$ or $p<0.001)$. In a word, it was demonstrated that L-menthol exerted antioxidant activity via reducing oxidative stress markers as well as enhancing the activities of antioxidants.

Effects of L-menthol on the protein expression of NF-KB p65, p38, p-p38, Nrf2 and NQO1

$\mathrm{NF}-\mathrm{\kappa B}$ behaves as a major regulator of cellular defences which is associated with inflammatory responses in many diseases. ${ }^{36}$
Nrf2 is a transcription factor which has played an important role in cellular redox balance. ${ }^{3}$ P38 subgroup of mitogenactivated protein kinases (MAPKs) can be activated by inflammation, oxidative stress and apoptosis, which are involved in chronic obstructive pulmonary disease. ${ }^{37}$ In this regards, the protein expressions of NF- $\kappa$ B p65, p38, p-p38, Nrf2 and NQO1 were evaluated using western blotting assays (Fig. 5). Quantitative densitometric analysis of NF- $\mathrm{B}$ p65, Nrf-2 and NQO1 proteins normalized against glyceraldehyde-3-phosphate dehydrogenase (GAPDH) whereas quantitative densitometric analyse of p-p38 was against p38.

Briefly, the expression levels of NF- $\mathrm{BB}$ p65 and p-p38 were enhanced in CSE group compared with blank control group $(p<$ 0.01 or $p<0.001)$. On the contrary, L-menthol down-regulated the expression of NF-KB p65 and p-p38 relative to CSE group $(p<0.05$ or $p<0.001)$. As for Nrf2 and NQO1, the expression levels were decreased after treated with CSE compared to blank control group $(p<0.001)$ whereas L-menthol significantly enhanced their expression $(p<0.001)$.

\section{Immunohistochemistry for the expression of MMP-9, TIMP-1,} $\mathrm{CD}^{+}$and $\mathrm{CD8}^{+}$cells

Protease-antiprotease imbalance has played a central role in the lung remodelling while the migration of $\mathrm{CD} 4^{+}$and $\mathrm{CD} 8^{+}$ cells promote destruction of lung tissues. ${ }^{2,38,39}$ Immunohistochemical staining was applied in this work for determining the expressions of MMP-9, TIMP- $1, \mathrm{CD}^{+}{ }^{+}$and $\mathrm{CD} 8^{+}$cells in lung of rats. Immunohistochemical staining lung tissue slices were observed under light microscope $(400 \times)$. Briefly, MMP-9, TIMP-1, CD4 ${ }^{+}$and $\mathrm{CD} 8^{+}$cells were abundantly found 

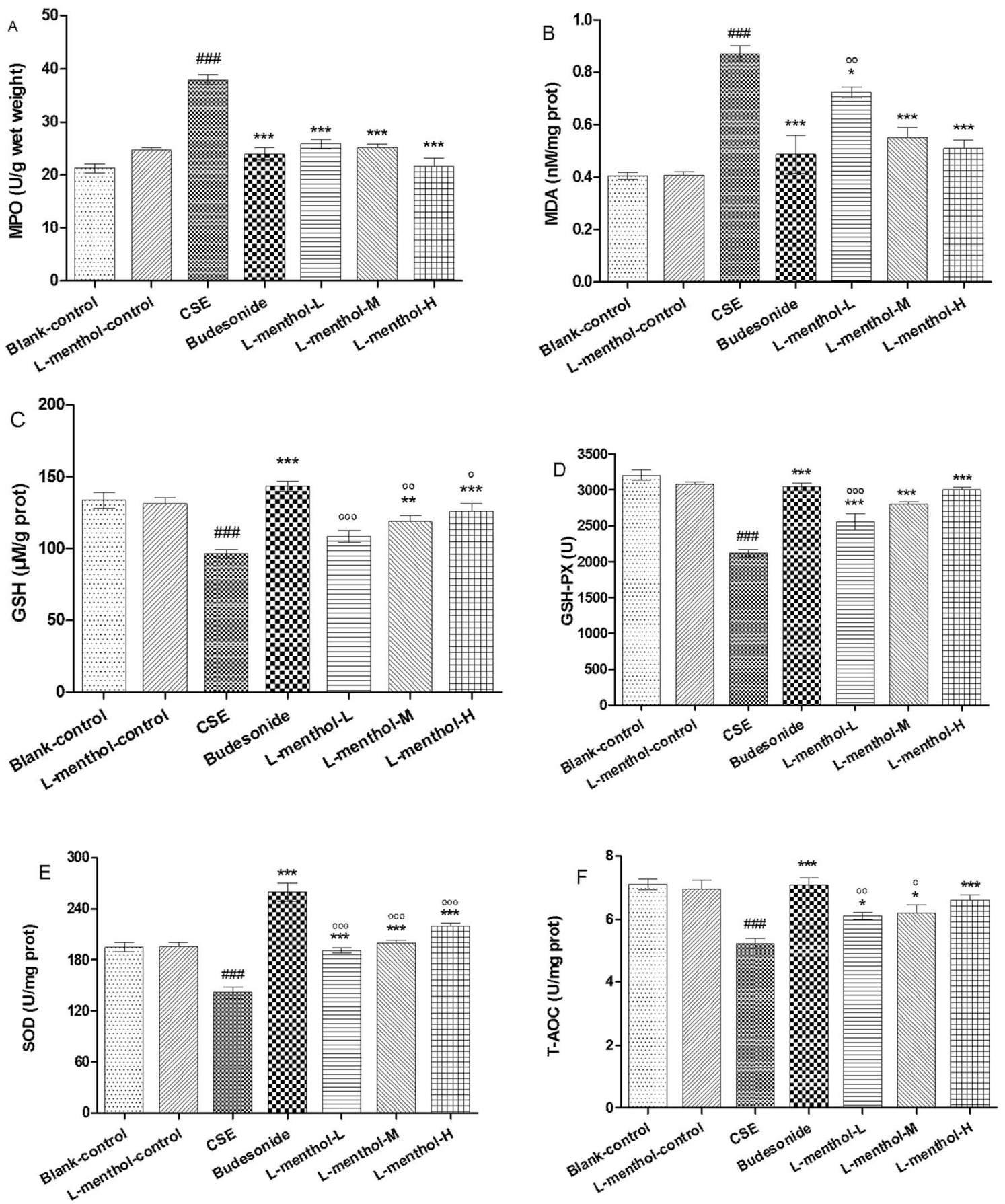

Fig. 4 Effect of L-menthol on myeloperoxidase (MPO) (A), malondialdehyde (MDA) (B), glutathione (GSH) (C), glutathione peroxidase (GSH-Px) (D), superoxide dismutase (SOD) (E) and total antioxidant capacity (T-AOC) (F). Data are presented as mean \pm SEM ( $n=6 /$ group). ${ }^{\#} p<0.05,{ }^{\# \#} p<$ 0.01 and ${ }^{\# \# \#} p<0.001$ vs. the blank control group. ${ }^{*} p<0.05$, ${ }^{* *} p<0.01$ and $* * * p<0.001$ vs. the CSE group. ${ }^{\circ} p<0.05,{ }^{\circ 0} p<0.01$ and ${ }^{\circ 00} p<0.001$ vs. the budesonide group.

in CSE group compare to blank control group whereas treated with L-menthol may reduce the expression level compared with CSE group (Fig. 6). In summary, these results support the idea that L-menthol might participate in the airway remodelling and immune-regulation in CSE-induced lung injury in rats.

\section{Discussion}

The reported pathogenesis of chronic obstructive pulmonary disease includes inflammation, oxidative stress and proteaseantiprotease imbalance while cigarette smoking is considered as the major reason. ${ }^{5}$ L-Menthol is the main ingredients in numbers of compound preparations which have been proved to 

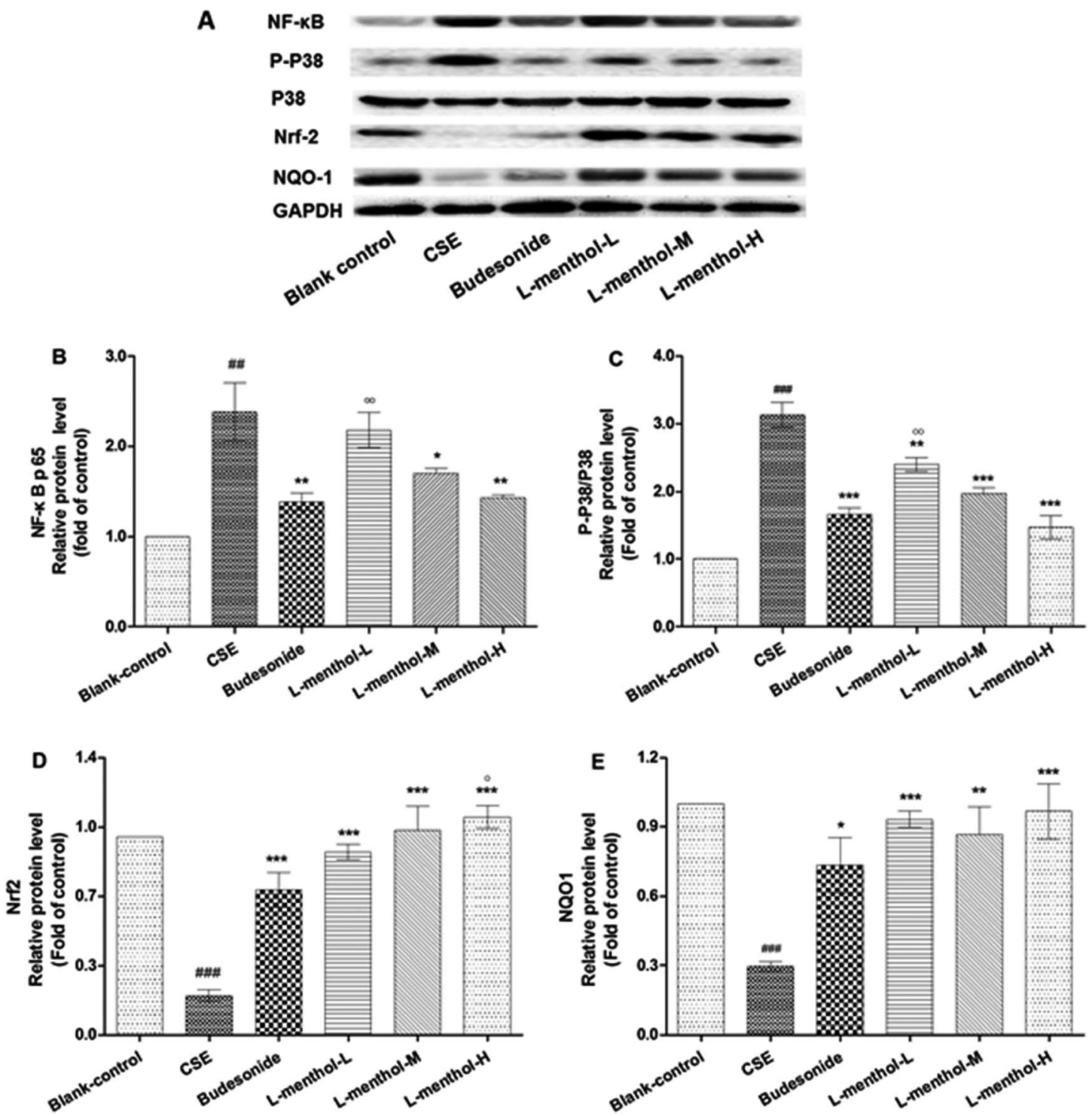

Fig. 5 The effect of L-menthol on the protein expression of nuclear factor kappa B p65 (NF- $\kappa B$ p65), p-p38, nuclear factor erythroid 2-related factor 2 (Nrf-2) and NAD(P)H: quinone oxidoreductase 1 (NQO1). Quantitative densitometric analyses of NF- $\mathrm{B}$ p65, Nrf-2 and NQO1 proteins normalized against glyceraldehyde-3-phosphate dehydrogenase (GAPDH) whereas quantitative densitometric analyse of $p$-p38 was against p38. Each value represents the mean \pm SEM of three independent experiments. ${ }^{\#} p<0.05,{ }^{\# \#} p<0.01$ and ${ }^{\# \# \#} p<0.001$ vs. the blank control group. ${ }^{*} p<0.05, * * p<0.01$ and ${ }^{* * *} p<0.001$ vs. the CSE group. ${ }^{\circ} p<0.05,{ }^{\circ} p<<0.01$ and ${ }^{\circ 00} p<0.001$ vs. the budesonide group.

be clinically effective in several respiratory system diseases. ${ }^{21-24,40}$ In this study, rat emphysema model was established in order to investigate the possible pharmacological effect of L-menthol. In summary, our results support that $\mathrm{L}^{-}$ menthol could alleviate CSE induced lung injury in rats by inhibiting oxidative stress and inflammation via nuclear factor kappa B, p38 MAPK and Nrf2 signalling pathways.

Previous studies demonstrated that inflammation has played an essential role which is involved in the development of COPD and many other respiratory diseases. Total white blood cell counts and differential count of macrophages, neutrophils and lymphocyte in BALF may partly reflect the airway inflammation. Macrophages are suggested to defence against noxious substances. Besides, macrophages and neutrophils are responsible for the production of pro-inflammatory cytokines such as TNF- $\alpha$, IL-1 $\beta$ and IL- $6 .^{41}$ TNF- $\alpha$ behaves as an essential pro-inflammatory cytokine which triggers a positive feedback loop during inflammation by the activation of NF- $\kappa$ B. IL-1 $\beta$ and IL-6 are important inflammatory mediators which were involved in lung inflammation, enlargement of distal airspaces and airway fibrosis. ${ }^{\mathbf{4 2 4 3}}$ In this work, it was revealed that L-menthol could significantly decrease the numbers of total white blood cells and macrophages toward normal level. For further evaluating the anti-inflammation activity of L-menthol, the levels of TNF- $\alpha$, IL- $1 \beta$ and IL- 6 in lung tissue were determined. Notably, the suppression of TNF- $\alpha$, IL- $1 \beta$ and IL- 6 were observed after treated with L-menthol. Taken together with the data, we hypothesized that L-menthol might diverse its anti- 


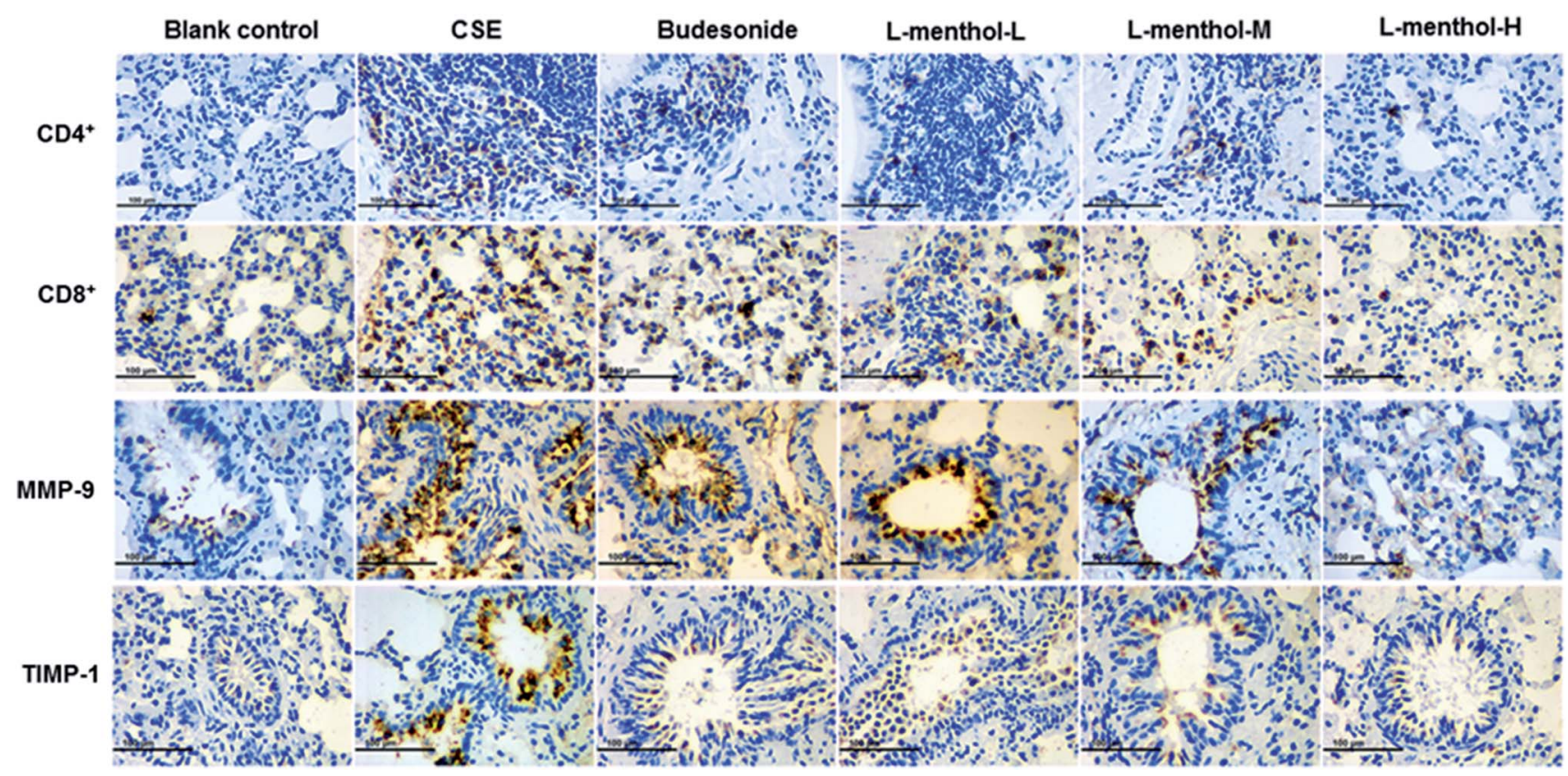

Fig. 6 Immunohistochemistry analysis of the effect of L-menthol on the expression of matrix metalloproteinase-9 (MMP-9), tissue inhibitor of metalloproteinases-1 (TIMP-1), CD4 ${ }^{+}$and $C D 8^{+}$T cells. Photomicrographs were taken at $400 x$

inflammation effects by reducing the amounts of total white blood cells and macrophages as well as suppressing proinflammation cytokines. Interestingly, this is in agreement with a previous work which demonstrated that L-menthol can exert inflammation activities. ${ }^{24}$

NF- $\mathrm{KB}$ is an important transcription factor which regulates number of genes involved in inflammation responses. In addition, inflammation and oxidative stress which are involved in COPD pathogenesis may activate the p38 subgroup of mitogen-activated protein kinases (MAPKs). ${ }^{37}$ Moreover, previous work revealed that $\mathrm{p} 38$ MAPK can be considered as a potential target for the treatment of COPD.$^{44}$ Our western blotting results showed that L-menthol suppressed the transcriptional activity of NF- $\kappa B$ and down-regulated of p38 MAPK pathways. This is consisted with a previous study which demonstrated that the expressions of NF- $\kappa \mathrm{B}$, Erk and p38 could be down-regulated by menthol administration. ${ }^{45}$

Oxidative stress is another essential element which is related to chronic inflammation. ${ }^{3}$ Therefore, in order to investigate the antioxidative activity of L-menthol on CSE induced lung injury, levels of antioxidant enzymes (SOD and GSH-Px) and contents of GSH, MDA and MPO as well as T-AOC were determined in this work. As known, MDA and MPO are important oxidative stress markers. Briefly, MDA, as a lipid peroxidation product, is an indicator of oxidative stress which is correlated inversely with pulmonary function. ${ }^{32}$ MPO can increase the amount of ROS along with other oxidase and finally leads to irreversible lung damage. ${ }^{33,34}$ Our results revealed that the MDA and MPO levels in lung were significantly decreased after treated with $\mathrm{L}^{-}$ menthol. Antioxidants can protect against the direct injurious effects of oxidants. ${ }^{33} \mathrm{GSH}$ is a key antioxidant for protecting airway epithelium from oxidant damage which can be converted into GSSG by GSH-Px, and has played an important role in the prevention of peroxidative lung damage in patients with COPD. ${ }^{35}$ SOD is a scavenger of superoxide radical in the body. ${ }^{33,35}$ In this study, it was suggested that the activities of the major scavenger enzymes (SOD and GSH-Px) and the content of GSH were significantly decreased whereas the levels of MDA and MPO were obviously elevated in rats from CSE group compared to blank control group. To our respect, the treatment of $\mathrm{L}^{-}$ menthol markedly increased the levels of SOD, GSH-Px and GSH while inhibited the production of MDA and MPO. Taken together, these results support the idea that $\mathrm{s}$-menthol could improve enzymatic and non-enzymatic antioxidant defence systems in order to relieve the oxidative damage induced by CSE.

Nrf2 protects against smoking triggered ROS through the transcription of target genes involved in redox homeostasis. NQO1 is a phase II detoxifying enzymes which can be regulated by Nrf2. ${ }^{3}$ In conclusion, our results revealed that the protein expression of Nrf2 and NQO1 were both markedly up-regulated in L-menthol groups compared with CSE group. It was demonstrated that the activation of Nrf2 might be responsible for the anti-oxidative effect of $\mathrm{L}$-menthol.

The imbalance between matrix metalloproteases (MMPs) and tissue inhibitors of MMPs (TIMPs) has played a pivotal role in the destruction of lung parenchyma and is related to the appearance of emphysema. ${ }^{46}$ In our work, an obvious alleviation of emphysema was observed in rats after treated with L-menthol through morphometric analysis. Moreover, the expression levels of MMP-9 and TIMP-1 in the lung were investigated using the immunohistochemical study. In line with expectations, significant higher expression levels of MMP-9 and TIMP-1 were observed in lungs of rats in CSE group compared with blank control group. Conversely, the expressions of MMP-9 and TIMP1 were strongly down-regulated by L-menthol compared to CSE 
group. In addition, cigarette smoking could induce chronic airway inflammation and subsequently lead to the excessive recruitment of $\mathrm{CD}^{+}$and $\mathrm{CD} 8^{+}$cells in the lungs. ${ }^{39,47}$ As known, $\mathrm{CD}^{+} \mathrm{T}$ cells exhibit cytotoxic activity toward antigens while $\mathrm{CD}^{+}{ }^{+} \mathrm{T}$ cells exert immune activity by recruiting and activating innate immune cells such as natural killer and macrophages independently. ${ }^{48,49}$ Regarding this, the immunohistochemical study was conducted so as to clarify the effect of L-menthol on the infiltration of $\mathrm{CD}^{+}$and $\mathrm{CD}^{+} \mathrm{T}$ cells in CSE treated rats. In conclusion, L-menthol could effectively suppress the infiltration of $\mathrm{CD}^{+}$and $\mathrm{CD} 8^{+} \mathrm{T}$ cells in lung tissues compared with CSE group.

Glucocorticoids such as budesonide have played a key role in the treatment of COPD, however, patients are suffering severe side effects including steroid diabetes, osteoporosis and glucocorticoid resistance. ${ }^{7}$ In our study, the body weights and spleen indexes in the budesonide group were significantly decreased compared with the blank control group whereas no changes were found in the L-menthol groups. Furthermore, rats in the L-menthol control group haven't shown any statistically significant difference in body weights, liver/spleen indexes, inflammation cytokines and oxidative stress biomarkers. Therefore, it might be concluded that L-menthol could be a safer agent in the treatment of COPD than budesonide.

\section{Conclusions}

In summary, L-menthol has shown an encouraging effect on CSE-induced lung injury in rats mainly by anti-inflammatory and anti-oxidative activities. Briefly, L-menthol exerted antiinflammatory effect through decreasing total white blood cells and macrophages influx as well as suppressing proinflammation cytokine production via inhibiting $\mathrm{NF}-\kappa \mathrm{B}$ and p38 MAPK pathways. Moreover, the mechanism by which Lmenthol expressed anti-oxidative activity can be concluded as reducing the levels of oxidative stress markers and enhancing the activities of antioxidant through Nrf2 pathway. Taken together with all the findings, L-menthol might be considered as a potential therapeutic drug for the treatment of COPD in future.

\section{Conflicts of interest}

There are no conflicts to declare.

\section{Acknowledgements}

This work was supported by the funds from National 'Major Science and Technology Project - Prevention and Treatment of AIDS, Viral Hepatitis, and Other Major Infectious Diseases' (Grant \#2013ZX10005004), 'Major Project of Science and Technology of Shandong Province' (Grant \#2015ZDJS04001), 'Science \& Technology Enterprise Technology Innovation Fund of Jiangsu Province' (Grant \#BC2014172), 'Small \& Medium Enterprise Technology Innovation Project of Lianyungang City' (Grant \#CK1333).

\section{References}

1 V. Kim and G. J. Criner, Am. J. Respir. Crit. Care Med., 2013, 187, 228-237.

2 P. Piesiak, A. Brzecka, M. Kosacka, E. Passowicz-Muszynska, T. Dyla and R. Jankowska, Pol. Merkur. Lekarski, 2011, 31, 270-273.

3 B. M. Fischer, J. A. Voynow and A. J. Ghio, Int. J. Chronic Obstruct. Pulm. Dis., 2015, 10, 261-276.

4 S. Sethi and T. F. Murphy, N. Engl. J. Med., 2008, 359, 23552365.

5 M. Miravitlles and J. J. Soler-Cataluna, Arch. Bronconeumol., 2017, 53, 89-90.

6 T. C. OShaughnessy, T. W. Ansari, N. C. Barnes and P. K. Jeffery, Am. J. Respir. Crit. Care Med., 1997, 155, 852-857.

7 Z. Ding, X. Li, Y. J. Lu, G. S. Rong, R. Q. Yang, R. X. Zhang, G. Q. Wang, X. Q. Wei, Y. Q. Ye, Z. X. Qian, H. Y. Liu, D. F. Zhu, R. Q. Zhou, K. Zhu, R. P. Ni, K. Xia, N. Luo and C. Pei, Respir. Med., 2016, 121, 39-47.

8 S. Nakagawa, H. Tagami and S. Aiba, Contact Dermatitis, 2009, 61, 178-179.

9 M. Emura and H. Matsuda, Chem. Biodiversity, 2014, 11, 1688-1699.

10 M. Nesterkina and I. Kravchenko, Nat. Prod. Commun., 2016, 11, 1419-1420.

11 H. O. Elansary, K. Yessoufou, S. Shokralla, E. A. Mahmoud and K. Skaicka-Wozniak, Ind. Crops Prod., 2016, 92, 50-56.

12 J. Plevkova, M. Kollarik, I. Poliacek, M. Brozmanova, L. Surdenikova, M. Tatar, N. Mori and B. J. Canning, J. Appl. Physiol., 2013, 115, 268-274.

13 E. A. Laude, A. H. Morice and T. J. Grattan, Pulm. Pharmacol., 1994, 7, 179-184.

14 M. G. Zaia, T. Cagnazzo, K. A. Feitosa, E. G. Soares, L. H. Faccioli, S. M. Allegretti, A. Afonso and F. Anibal Fde, Front. Pharmacol., 2016, 7, 170.

15 A. L. Rozza, F. Meira de Faria, A. R. Souza Brito and C. H. Pellizzon, PLoS One, 2014, 9, e86686.

16 N. Mimica-Dukic, B. Bozin, M. Sokovic, B. Mihajlovic and M. Matavulj, Planta Med., 2003, 69, 413-419.

17 J. Suchodolski, J. Feder-Kubis and A. Krasowska, Microbiol. Res., 2017, 197, 56-64.

18 U. R. Juergens, M. Stober and H. Vetter, Eur. J. Med.Res., 1998, 3, 539-545.

19 C. Mogosan, O. Vostinaru, R. Oprean, C. Heghes, L. Filip, G. Balica and R. I. Moldovan, Molecules, 2017, 22, 1-11.

20 T. Nishino, Y. Tagaito and Y. Sakurai, Am. J. Respir. Crit. Care Med., 1997, 156, 309-313.

21 J. Y. XIaoshan Zhang and G. Zhang, Lishizhen Med. Mater. Med. Res., 2008, 19, 678-679.

22 R. Hao and H. Nie, Shaanxi J. Tradit. Chin. Med., 2010, 31, 1625-1626.

23 H. He, J. Guiyang Med. Coll., 2016, 41, 615-617.

24 Z. L. BAI Yang, M. A. Zang and L. I. Juan, Pharmacol. Clin. Chin. Mater. Med., 2013, 4, 46-49.

25 Z. H. He, P. Chen, Y. Chen, S. D. He, J. R. Ye, H. L. Zhang and J. Cao, Tob. Induced Dis., 2015, 13, 1-8. 
26 M. Deng, J. Li, Y. Gan, Y. Chen and P. Chen, Int. J. Chronic Obstruct. Pulm. Dis., 2017, 12, 861-872.

27 Y. Zhang, J. Cao, Y. Chen, P. Chen, H. Peng, S. Cai, H. Luo and S. J. Wu, Exp. Lung Res., 2013, 39, 18-31.

28 K. H. Choe, L. Taraseviciene-Stewart, R. Scerbavicius, L. Gera, R. M. Tuder and N. F. Voelkel, Am. J. Respir. Crit. Care Med., 2003, 167, 1516-1521.

29 M. Saetta, R. J. Shiner, G. E. Angus, W. D. Kim, N. S. Wang, M. King, H. Ghezzo and M. G. Cosio, Am. Rev. Respir. Dis., 1985, 131, 764-769.

30 X. Zhu, X. Jiang, A. Li, Y. Sun, Y. Liu, X. Sun, X. Feng, S. Li and Z. Zhao, Biochem. Biophys. Res. Commun., 2017, 491, 821-826.

31 P. Castro, A. Legora-Machado, L. Cardilo-Reis, S. Valenca, L. C. Porto, C. Walker, C. Zuany-Amorim and V. L. G. Koatz, Eur. J. Pharmacol., 2004, 498, 279-286.

32 Z. Kluchova, D. Petrasova, P. Joppa, Z. Dorkova and R. Tkacova, Physiol. Res., 2007, 56, 51-56.

33 W. MacNee, Chest, 2000, 117, 303S-317S.

34 I. Rahman, Cell Biochem. Biophys., 2005, 43, 167-188.

35 R. R. Rai and M. S. Phadke, Indian J. Clin. Biochem., 2006, 21, 161-164.

36 J. W. Rhee, K. W. Lee, D. Kim, Y. Lee, O. H. Jeon, H. J. Kwon and D. S. Kim, J. Biochem. Mol. Biol., 2007, 40, 88-94.

37 T. Renda, S. Baraldo, G. Pelaia, E. Bazzan, G. Turato, A. Papi, P. Maestrelli, R. Maselli, A. Vatrella, L. M. Fabbri, R. Zuin, S. A. Marsico and M. Saetta, Eur. Respir. J., 2008, 31, 62-69. 38 J. L. Curtis, C. M. Freeman and J. C. Hogg, Proc. Am. Thorac. Soc., 2007, 4, 512-521.
39 E. Roos-Engstrand, B. Ekstrand-Hammarstrom, J. Pourazar, A. F. Behndig, A. Bucht and A. Blomberg, COPD, 2009, 6, 112-120.

40 Y. Z. Yilu Chen, J. Zhou, Y. Huang and S. Li, China Pharm., 2003, 14, 726-727.

41 S. D. Shapiro, Am. J. Respir. Crit. Care Med., 1999, 160, S29S32.

42 M. L. Ji, Y. X. Wang, X. P. Li and Z. B. Qian, Int. J. Clin. Exp. Med., 2015, 8, 21956-21963.

43 U. Lappalainen, J. A. Whitsett, S. E. Wert, J. W. Tichelaar and K. Bry, Am. J. Respir. Cell Mol. Biol., 2005, 32, 311-318.

44 C. Huang, M. Xie, X. He and H. Gao, Med. Sci. Monit., 2013, 19, 1229-1235.

45 Z. Liu, C. Shen, Y. Tao, S. Wang, Z. Wei, Y. Cao, H. Wu, F. Fan, C. Lin, Y. Shan, P. Zhu, L. Sun, C. Chen, A. Wang, S. Zheng and Y. Lu, Food Chem. Toxicol., 2015, 82, 12-18.

46 E. Mocchegiani, R. Giacconi and L. Costarelli, Curr. Opin. Pulm. Med., 2011, 17(suppl. 1), S11-S19.

47 S. Zhuo, N. Li, Y. Zheng, X. Peng, A. Xu and Y. Ge, Cell Biochem. Biophys., 2015, 73, 505-511.

48 J. Jackute, M. Zemaitis, D. Pranys, B. Sitkauskiene, S. Miliauskas, V. Bajoriunas and R. Sakalauskas, Medicina (Kaunas), 2015, 51, 263-271.

49 Y. Godet, E. Fabre, M. Dosset, M. Lamuraglia, E. Levionnois, P. Ravel, N. Benhamouda, A. Cazes, F. Le Pimpec-Barthes, B. Gaugler, P. Langlade-Demoyen, X. Pivot, P. Saas, B. Maillere, E. Tartour, C. Borg and O. Adotevi, Clin. Cancer Res., 2012, 18, 2943-2953. 